\title{
Genetic analysis and clinical assessment of four patients with Glycogen Storage Disease Type Illa in China
}

\author{
Yu Zhang ${ }^{1}$, Mingming Xu', Xiaoxia Chen ${ }^{1}$, Aijuan Yan ${ }^{1}$, Guoyong Zhang ${ }^{1}$, Zhenguo Liu ${ }^{1 *}$ (D) and Wenjuan Qiu ${ }^{2 *}$
}

\begin{abstract}
Background: Glycogen Storage Disease Type III (GSD III) is a rare autosomal recessive metabolic disorder caused by AGL gene mutation. There is significant heterogeneity between the clinical manifestations and the gene mutation of AGL among different ethnic groups. However, GSD III is rarely reported in Chinese population.

Case presentation: In this study, we aimed to study the genetic and clinical characteristics of four patients with GSD Illa from China, especially the neurological manifestations. Meanwhile, we conducted a literature review of GSD Illa cases reported in Chinese population to investigate the relationship between genotype and phenotype.

Conclusions: Three different AGL gene mutations were identified in our patients: c.206dupA, c.1735 + 1G > T and c. 2590 C > T. Moreover, progressive myopathy accompanied by elevated creatine kinase level was the main manifestation of our patients in adolescents. Our results showed that AGL c.206dupA was a novel mutation and caused severe clinical manifestations. AGL c.1735 + 1G > T might be a recurrent mutation in the Chinese population. Genetic analysis of $A G L$ gene mutation combined with muscle magnetic resonance imaging (MRI) might provide greater benefit to the patient in diagnosing GSD IIla, rather than an invasive diagnostic procedure of biopsy.
\end{abstract}

Keywords: Glycogen storage disease Illa, AGL gene, Clinical characteristics, Chinese

\section{Background}

Glycogen Storage Disease Type III (GSD III) is a rare autosomal recessive metabolic disorder. There are two major clinical types of GSD III. GSD IIIa involves liver and muscle while GSD IIIb only affects the liver. GSD III commonly presents with growth retardation, fasting hypoglycemia, hepatomegaly, and seizures during childhood. The neuromuscular manifestations of GSD IIIa are mainly reported in adults [1-3]. GSD III is caused by $A G L$ gene mutation leading a deficiency of glycogen debrancher enzyme activity. The human $A G L$ gene is located on chromosome $1 \mathrm{p} 21$ and consists of 35 exons spanning $\sim 85 \mathrm{~kb}$ of genomic DNA. $A G L$ gene is expressed from the third exon [4]. Genetic analysis of

\footnotetext{
*Correspondence: liuzhenguo@xinhuamed.com.cn;

qiuwenjuan@xinhuamed.com.cn

'Department of Neurology, Xinhua Hospital Affiliated to Shanghai Jiao Tong University School of Medicine, 1665 Kong jiang Road, Shanghai 200092, People's Republic of China

${ }^{2}$ Department of Pediatric Endocrinology/Genetics, Shanghai Institute for Pediatric Research, Xinhua Hospital Affiliated to Shanghai Jiao Tong University School of Medicine, Shanghai 200092, China
}

the $A G L$ gene in several ethnic populations has revealed over 150 different $A G L$ gene mutations [5]. However, few $A G L$ mutations have been reported in the Chinese population in mainland China. In this study, we reported four Chinese patients with GSD IIIa and analyzed the relationship between genotype and phenotype of GSD IIIa in the Chinese population.

\section{Case presentation}

Our study included three male and one female GSD IIIa Chinese patients from three families. Patients 3 and 4 were siblings from one family. When these patients were admitted to our hospital, their clinical information, such as age, gender, height, weight, liver function and clinical symptoms were collected. Then, routine laboratory test, echocardiography, electromyogram, abdominal ultrasound, muscle MRI and neuropsychological test were conducted. All of this clinical information of the first visit and two years follow-up were shown in Table 1 . The guideline for the diagnosis of GSD III had been followed [5]. 
Table 1 Clinical features and gene mutation of GSD IIla patients

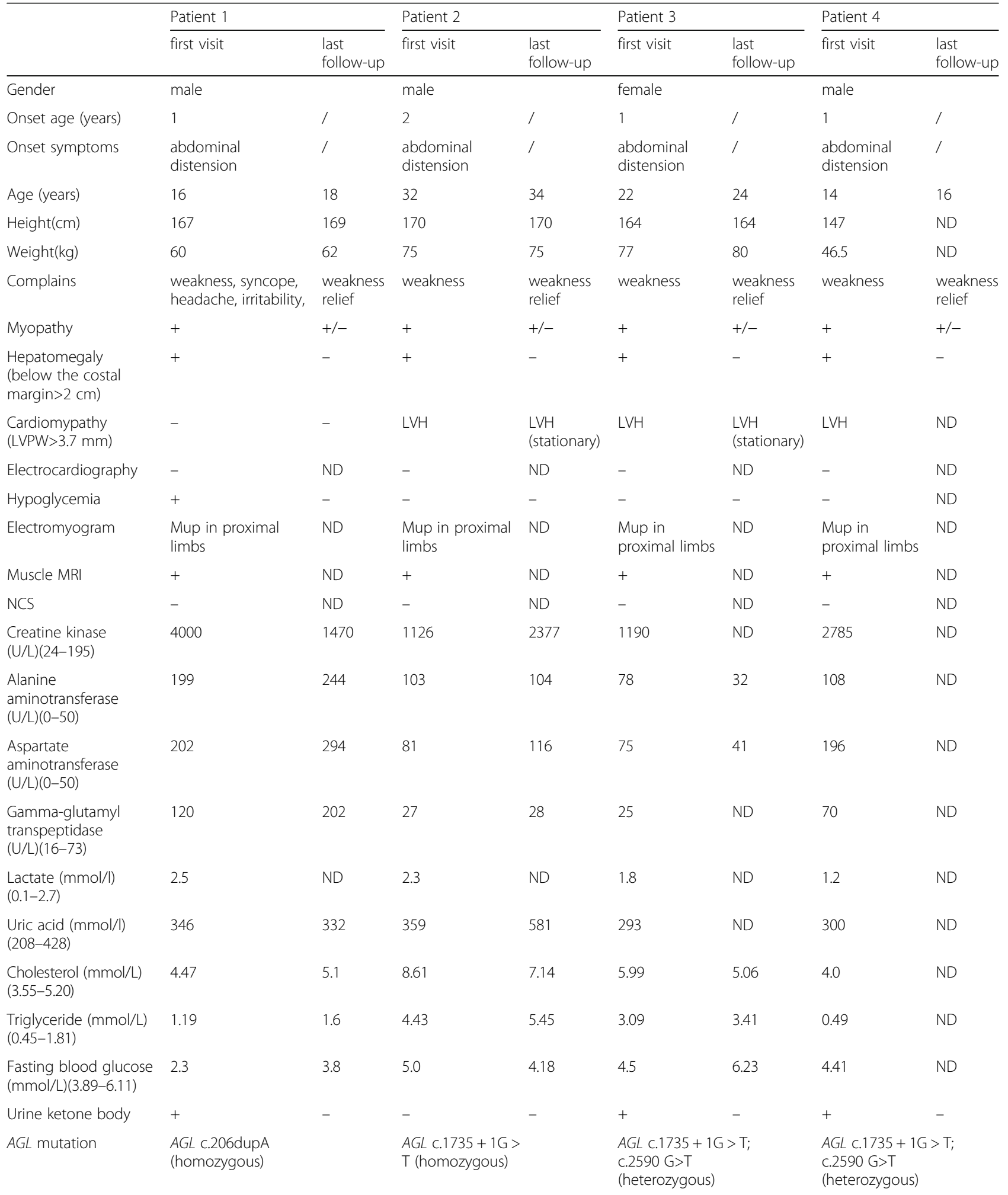

LVPW Left Ventricular Posterior Wall, LVH Left ventricular hypertrophy, MUP Motor unit potential, NCS Nerve Conduction Studies, ND not done

Our patients mainly complained physical weakness accompanied by elevated creatine kinase level. Although patients had not received high-protein and uncooked cornstarch (UCS) diet therapy in childhood, growth retardation was not observed after adolescence. Treatment with high-protein and UCS diet was implemented in all 
patients after the first visit, according to preprandial blood glucose and the level of abnormal alanine aminotransferase (ALT) and aspartate aminotransferase (AST). After high-protein and UCS diet therapy, clinical manifestations of weakness of our patients were significantly relieved at the last follow-up. Currently, their therapy followed standard treatment guidelines, with UCS in $1.5 \mathrm{~g} / \mathrm{kg} /$ time for $1-3$ times per day and protein in $2 \mathrm{~g} / \mathrm{kg} / \mathrm{d}$.

The results of laboratory tests showed the level of creatine kinase $(\mathrm{CK})$ was significantly increased among all patients, but ketonic hypoglycemia was only detected in patient 1 . Serum cholesterol and triglyceride were normal in patient 1 but they were elevated in other patients. After a high-protein and UCS diet therapy for two years, the level of CK was still 5-10 times over the upper limit of normal, but preprandial blood glucose, serum cholesterol and triglyceride of all patients had nearly normalized at the last follow-up.

All our patients showed asymptomatic cardiac hypertrophy accompanied by normal ejection fraction and cardiac conduction (Table 1). Meanwhile, hepatomegaly accompanied by abnormal ALT and AST was common during our patients' childhood. After a high-protein and UCS diet therapy for two years, cardiac hypertrophy of our patients did not deteriorated. Moreover, levels of AST and ALT had decreased and hepatic adenoma was not detected in our patients. We didn't observe hepatic cirrhosis or hepatocellular carcinoma in our patients by liver ultrasound or laboratory tests. Indeed, we did not perform the liver biopsy which was invasive and potentially detrimental to the patients.

To better identify the neurological manifestations of GSD IIIa in our patients, nerve conduction studies (NCS), electromyogram (EMG), muscle MRI, brain MRI, electroencephalogram and neuropsychological tests were performed. All patients showed normal results in NCS, brain MRI and electroencephalogram, but the results of EMG were abnormal. Myopathic motor unit potential was detected in all patients, but the specificity and sensitivity were not satisfactory. Notably, Muscle MRI transverse sections from the mid-thigh and lower leg muscles of patient 2 presented mild T1 signal intensity in the long head of femoris and peroneus longus muscles (arrows), indicating an increase in adipose-like tissue (Fig. 1).What's more, lateral heads of gastrocnemius muscles showed atrophy in patient 2 (Fig. 1). In general, the posterior and lateral muscles of the lower limbs were mostly affected (Fig. 1).

\section{DNA sequence analysis of $A G L$ gene}

The genomic DNA of all family members was extracted from peripheral blood by using the standard phenolchloroform extraction method. Polymerase chain reaction using the primers located in the flanking introns was conducted to amplify the $A G L$ gene. $A G L$ gene mutations were screened by direct sequencing using an $\mathrm{ABI}$ Prism 3100 Genetic Analyzer (Applied Biosystems, USA). The sample sequences were compared with the genomic DNA sequence of $A G L$ (GenBank accession no. NM_000642.2).

We found three different $A G L$ mutations (Fig. 2) in our four patients with GSD IIIa from three families. Among these three mutations, one mutation was novel and the other two were reported. Patient 1 , who still had frequent episodes of hypoglycemia in adolescence, carried a homozygous insert mutation $A G L$ c.206dupA (p. N69Kfs*8). This $A G L$ c.206dupA mutation is a frameshift mutation causing premature termination codons in the exon 4 and is predicted to be "disease-causing mutation" by MutationTaster (http://www.mutationtaster. org/).The AGL c.206dupA mutation has not been documented in any public database, nor in our internal exonic database of OMIM genes from 1000 individuals. Patient 2 was homozygous for the $A G L$ c. $1735+1 \mathrm{G}>$ T (IVS14 + 1G>T) splice site mutation, which was predicted to impair normal splicing. For patients 1 and 2, the homozygous mutations were inherited from their parents separately. Patients 3 and 4 had
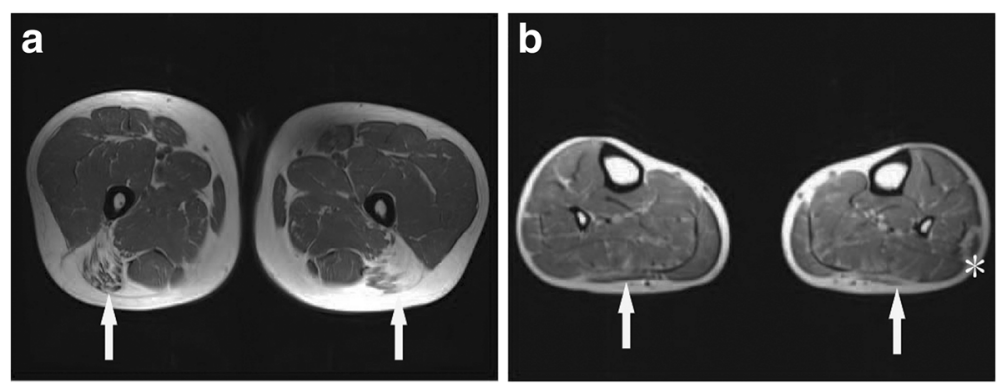

Fig. 1 Muscle MRI comprising T1-weighted images of the thigh and lower leg from patient 2. a. Transverse cuts from the mid-thigh muscles showed mild increase in signal intensity within the femoris long head (arrows); $\mathbf{b}$, Transverse cuts from the lower leg showed mild increase in signal intensity within the peroneus longus (arrows) and there was atrophy of gastrocnemius lateral heads (asterisk) 
a

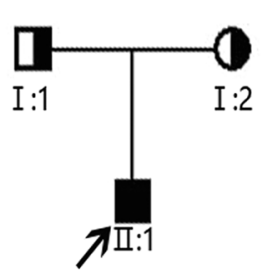

b

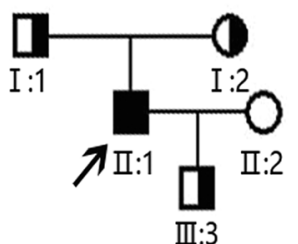

C

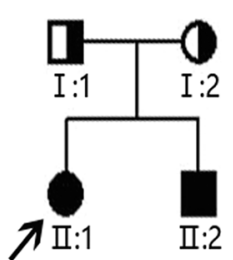

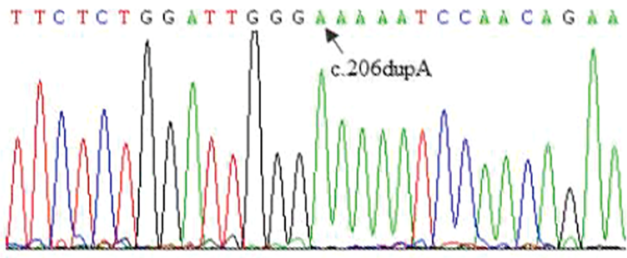

T T C C T TA A TA A A G G TAG GCT T G T T G G A G
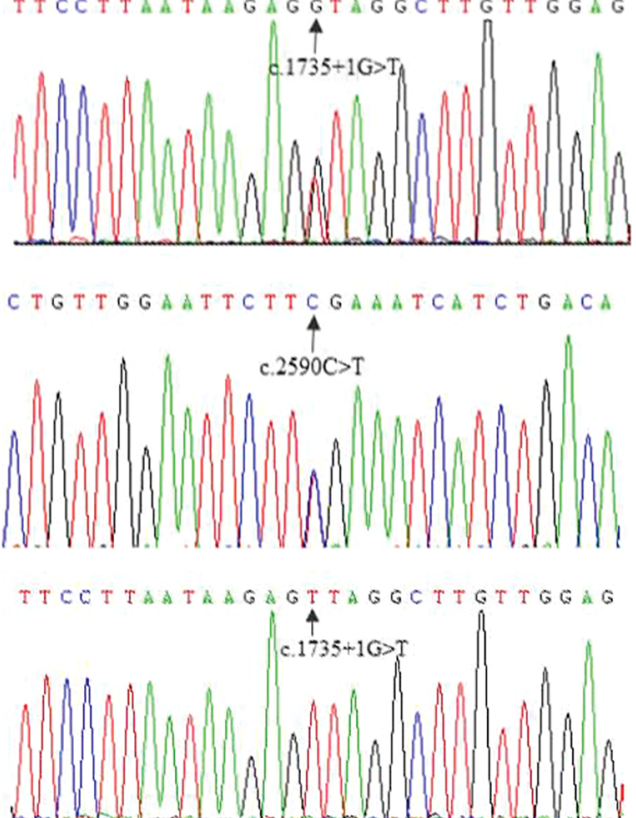

Fig. 2 Pedigrees of our patients with GSD IIla and mutation analysis of AGL gene. Filled circles and squares represent affected females and males, respectively. Proband is indicated with an arrow; a. patient 1 (II:1) from family 1. The DNA sequence chromatogram of the proband indicates AGL c.206dupA homozygous mutation indicated with an arrow; $\mathbf{b}$. patient 2 (II:1) from family 2 . The DNA sequence chromatogram of the proband indicates AGL c.1735 + 1G > T homozygous mutation with an arrow; c. patient $3(\|: 1)$ and patient 4 (II:2) from family 3 . The DNA sequence chromatogram of the proband indicates AGL C.1735 + 1G > T and AGL C.2590 C>T compound heterozygous mutations indicated with arrows

compound heterozygous mutations. One of the mutations is $A G L$ c.2590 $\mathrm{C}>\mathrm{T}$ which is inherited from their mother. This mutation results in substitution of arginine at codon 864 by termination codon (p. $\mathrm{R} 864 \mathrm{X}$ ) and causes premature termination. Another mutation is $A G L$ c. $1735+1 G>\mathrm{T}$ which is inherited from their father.

\section{Literature review}

Literature review of GSD IIIa reported in Chinese patients was conducted by searching for studies published from 1996 to 2017, with the keywords "Glycogen Storage Disease Type III", "glycogen debranching enzyme" and "AGL gene".The databases included Pub Med, Medline, VIP database and Chinese Biology Medicine. Only studies published in English or Chinese were included. All relevant papers were read carefully.

We had reviewed five papers including eighteen cases of GSD IIIa Chinese patients [6-10]. The detailed clinical information of these patients were shown in
Additional file 1: Table S1. There was fifteen male and three female cases. The mean age was $5.7 \pm 10.3$ years old (median 4 years; range 1-46 years). Hepatomegaly and myopathy were common clinical manifestations in Chinese GSD IIIa patients on different stages of individual development, but hepatic adenoma was rarely reported. CK, ALT, AST, cholesterol, triglyceride and fasting blood-glucose were significantly abnormal in GSD IIIa patients in China. A variety of mutations of the $A G L$ gene were found in Chinese GSD IIIa patients, including deletion, missense, nonsense and splicing mutations. There were five patients with $A G L$ c. $1735+1 \mathrm{G}>\mathrm{T}$ mutation. Therefore, $A G L$ c. $1735+1 \mathrm{G}>\mathrm{T}$ might be the most recurrent mutation in Chinese patients.

\section{Discussion and conclusions}

GSD III is caused by mutations in the $A G L$ gene. Over 150 different mutations in the $A G L$ gene have been identified. However, few $A G L$ mutations have been reported in mainland China $[4,5]$. Genetic analysis of our four 
Chinese GSD IIIa patients revealed three different mutations in $A G L$ gene. One novel frameshift insert mutation c.206dupA (N69Kfs*8) is located in exon4. It is likely be classified as a pathogenic mutation by following the ACMG/AMP sequence variant pathogenicity classification guideline [11]. As to the relationship between genotype and phenotype, we found the patient with $A G L \mathrm{c}$. 206dupA mutation showed severe clinical manifestations compared with the other patients. The patient carrying the AGL c.206dupA mutation still suffered severe hypoglycemia symptoms even in adolescence, which were absent in other patients. These manifestations indicated $A G L$ c.206dupA mutation might cause more severe functional deficiency of the glycogen debranching enzyme than other mutations. Therefore, the patient with $A G L$ c.206dupA mutation required a high-protein and UCS diet therapy even in adults. Then, AGL c. $1735+1 \mathrm{G}>\mathrm{T}$ mutation, which is first reported in Japan [12], is predicted to impair normal splicing. $A G L$ c. $1735+1 G>T$ mutation might be a recurrent mutation in Chinese patients and should be given priority when gene detection is conducted in Chinese GSD IIIa patients. Moreover, AGL c.2590 C>T,which is first reported by Caucasians [13], is predicted to lead to premature termination, which completely abolishes the enzyme activity. In summary, the patient carrying AGL c.206dupA homozygous mutation suffered more severe disease, whereas patient 2 carrying the splicing homozygous mutation had milder disease. This observation is in keeping with the notion that some residual functional protein may be produced from the splicing mutation.

We have collected detailed clinical information of four Chinese GSD IIIa patients from three families. We revealed clinic characteristics of Chinese GSD IIIa: Firstly, all of our patients mainly complained of weakness when running and climbing stairs, and a high-protein and UCS diet therapy could improve the symptom of muscle weakness effectively. Secondly, our patients showed growth retardation in childhood, but they can catch up to normal level in adolescence. Mental or physical development disabilities did not appear in most of patients. Thirdly, asymptomatic hepatomegaly is common in our patients. Liver and cardiac dysfunction is not significant in all of our patients. Finally, our study showed muscle MRI combined with genetic testing might be reliable, convenient and less invasive compared with muscle biopsy $[14,15]$.

In conclusion, our study provides a comprehensive overview of genetic and clinical features of GSD IIIa in China. Our results revealed that muscle weakness accompanied with high level of CK are the main clinical manifestations in adolescence. Cardiomyopathy and hepatic adenoma needs long term follow-up. $A G L$ c.206dupA mutation is a novel mutation leading severe clinical manifestations and AGL C.1735 + 1G > T might be a recurrent mutation in Chinese patients with GSD IIIa. Moreover, clinical history, muscle MRI and genetic testing may provide greater benefit to the patient in diagnosing GSD IIIa.

\section{Additional file}

Additional file 1: Table S1. Clinical and genetic features of GSD IIla patients reported in China. Literature review of GSD IIla reported in Chinese patients was conducted by searching for studies published from 1996 to 2017. (DOCX $17 \mathrm{~kb}$ )

\section{Abbreviations}

EMG: Electromyogram;; GSD III: Glycogen storage disease type III; NCS: Nerve conduction studies; UCS: Uncooked cornstarch

\section{Acknowledgments}

We thank all patients for participating in this study.

\section{Funding}

This study was supported by grants from the National Natural Science Foundation of China (81301081,81170811, 30973216), Shanghai Municipal Commission of Health and Family Planning Foundation (20134005), the National key R\&D Program of China (2017YFC1310300, 2016YFC0905100).

Availability of data and materials

All data generated or analysed during this study are included in this published article.

\section{Authors'contributions}

YZ analyzed the patient data and written the manuscript. MX, XC, GZ and AY are responsible for collecting patient data. ZL and WQ are responsible for research designs. All authors read and approved the final manuscript

\section{Ethics approval and consent to participate}

This study was performed with the approval of the Ethics Committee of Xinhua Hospital affiliated to Shanghai Jiao Tong University School of Medicine.

\section{Consent for publication}

Consent Form for Publication of personal information in a scientific journal, including clinical data and image and photographs was obtained from all individual participants included in the study or from their parents in the case of minors.

\section{Competing interests}

The authors declare that they have no competing interests.

\section{Publisher's Note}

Springer Nature remains neutral with regard to jurisdictional claims in published maps and institutional affiliations.

Received: 26 November 2017 Accepted: 19 March 2018

Published online: 04 April 2018

References

1. Shen JJ, Chen YT. Molecular characterization of glycogen storage disease type III. Curr Mol Med. 2002;2:167-75.

2. Shin YS. Glycogen storage disease: clinical, biochemical, and molecular heterogeneity. Semin Pediatr Neurol. 2006;13:115-20.

3. Kishnani PS, Austin SL, Arn P, Bali DS, Boney A, Case LE, Chung WK, Desai DM, El-Gharbawy A, Haller R, et al. Glycogen storage disease type III diagnosis and management guidelines. Genet Med. 2010;12:446-63.

4. Yang-Feng TL, Zheng K, Yu J, Yang BZ, Chen YT, Kao FT. Assignment of the human glycogen debranching gene to chromosome 1p21. Genomics. 1992; 13:931-4.

5. Goldstein JL, Austin SL, Boyette K, Kanaly A, Veerapandiyan A, Rehder C, Kishnani PS, Bali DS. Molecular analysis of the AGL gene: identification of 25 novel mutations and evidence of genetic heterogeneity in patients with glycogen storage disease type III. Genet Med. 2010;12:424-30. 
6. Yu X, Pan K, Huang Y, Zhang J. Four cases of the glycogen storage diseases. J Clin Pediatr. 2009;10:987-8.

7. Qiu Z, Huang W, Wei M, Qiu J, Zhang H, Wu X. Mutation analysis of AGL gene in 7 Chinese patients with glycogen storage disease type III. Basic \& Clinical Medicine. 2011;31:471-4.

8. Wu X, Pan J, Guo Y. AGL gene analysis of a pedigree with glycogen storage disease type III and identification of a novel mutation. Chin J Pediatr. 2013; 51:915-20

9. Chen X, Niu H, Yan W, He N, Cheng S. Clinical manifestation, pathological feature and treatment of 14 cases of glycogen storage disease. J Development Med. 2014;2:224-7.

10. Guo L, Lin W, Zhang Z, Zhao X, Zhang S, Cai X, Zhou Q, Song Y. Analysis of clinical features and AGL gene mutations in a family with glycogen storage disease type IIla. Clin. J Med Genet. 2015;32:502-5.

11. Richards S, Aziz N, Bale S, Bick D, Das S, Gastier-Foster J, Grody WW, Hegde M, Lyon E, Spector E, et al. Standards and guidelines for the interpretation of sequence variants: a joint consensus recommendation of the American College of Medical Genetics and Genomics and the Association for Molecular Pathology. Genet Med. 2015;17:405-24.

12. Horinishi A, Okubo M, Tang NL, Hui J, To KF, Mabuchi T, Okada T, Mabuchi $H$, Murase T. Mutational and haplotype analysis of AGL in patients with glycogen storage disease type III. J Hum Genet. 2002;47:55-9.

13. Endo $Y$, Hofinishi A, Vorgerd M, Aoyama Y, Ebara T, Murase T, Odawara M, Podskarbi T, Shin YS, Okubo M. Molecular analysis of the AGL gene: heterogeneity of mutations in patients with glycogen storage disease type III from Germany, Canada, Afghanistan, Iran, and Turkey. J Hum Genet. 2006; 51:958-63.

14. Preisler N, Laforêt P, Madsen KL, Prahm KP, Hedermann G, Vissing CR, Galbo $\mathrm{H}$, Vissing J. Muscle metabolism is impaired during exercise in glycogen storage disease type III. Neurology. 2015;84:1767-71.

15. Preisler N, Pradel A, Husu E, Madsen KL, Becquemin MH, Mollet A, Labrune $P$, Petit $F$, Hogrel JY, Jardel $C$, et al. Exercise intolerance in glycogen storage disease type III: weakness or energy deficiency? Mol Genet Metab. 2013;109: $14-20$.

\section{Submit your next manuscript to BioMed Central and we will help you at every step:}

- We accept pre-submission inquiries

- Our selector tool helps you to find the most relevant journal

- We provide round the clock customer support

- Convenient online submission

- Thorough peer review

- Inclusion in PubMed and all major indexing services

- Maximum visibility for your research

Submit your manuscript at www.biomedcentral.com/submit

) Biomed Central 